\title{
Liquid-liquid equilibria of aqueous mixtures containing selected dibasic esters and/or methanol
}

\author{
Shih-Bo Hung ${ }^{a}$, Ho-Mu Lin ${ }^{a}$, Cheng-Ching Yu ${ }^{b}$, \\ Hsiao-Ping Huang ${ }^{b}$, Ming-Jer Lee ${ }^{\mathrm{a}, *}$ \\ ${ }^{a}$ Department of Chemical Engineering, National Taiwan University of Science and Technology, \\ 43 Keelung Road, Section 4, Taipei 106-07, Taiwan \\ ${ }^{\mathrm{b}}$ Department of Chemical Engineering, National Taiwan University, 1 Roosevelt Road, \\ Section 4, Taipei 106-17, Taiwan
}

Received 8 June 2006; received in revised form 1 August 2006; accepted 1 August 2006 Available online 5 August 2006

\begin{abstract}
The liquid-liquid equilibrium (LLE) properties were measured for the ternary systems of water + methanol + dimethyl adipate, water + adipic acid monomethyl ester + dimethyl adipate and water + methanol + dimethyl glutarate at temperatures ranging from 298.15 to $318.15 \mathrm{~K}$ under atmospheric pressure. Each ternary system exhibited type-I LLE behavior and the region of heterogeneity was found to increase with a decrease of temperature. These new LLE data were correlated with the NRTL model and also used to examine the validity of various versions of the UNIFAC models. (C) 2006 Elsevier B.V. All rights reserved.
\end{abstract}

Keywords: LLE; Water; Dibasic esters; Methanol; NRTL; UNIFAC

\section{Introduction}

Dimethyl adipate (DMA), dimethyl glutarate (DMG) and dimethyl succinate (DMS) are the major components in dibasic esters (DBE) blends, which are widely used as paint strippers. DBE have also been suggested as potential green solvents to replace conventional high volatility and high toxicity organic solvents in industrial applications [1,2]. DBE can be synthesized from dibasic acids with alcohols via esterification, and the dibasic acids, such as adipic acid and glutaric acid, are available from caprolactam plants, in which the dibasic acids are considered as waste compounds in the intermediate streams. These process wastes could be feasibly recovered with reactive distillation technology. In the reactive distillation process unit, it involves several reversible reactions. For example, the esterification of adipic acid (AA) with methanol (MeOH) includes two reversible cascade reactions with an intermediate of adipic acid

\footnotetext{
* Corresponding author. Tel.: +88622737 6626; fax: +886227376644.

E-mail address: mjl@ch.ntust.edu.tw (M.-J. Lee).
}

monomethyl ester (or monomethyl adipate, MMA):

$$
\begin{aligned}
& \underset{(\mathrm{AA})}{\mathrm{C}_{4} \mathrm{H}_{8}(\mathrm{COOH})_{2}}+\underset{(\mathrm{MeOH})}{\mathrm{CH}_{3} \mathrm{OH}} \Leftrightarrow \underset{(\mathrm{MMA})}{(\mathrm{COOH}) \mathrm{C}_{4} \mathrm{H}_{8}\left(\mathrm{CH}_{3} \mathrm{COO}\right)+\mathrm{H}_{2} \mathrm{O} ;} \\
& \underset{(\mathrm{COOH}) \mathrm{C}_{4} \mathrm{H}_{8}\left(\mathrm{CH}_{3} \mathrm{COO}\right)}{(\mathrm{MMA})}+\underset{(\mathrm{MeOH})}{\mathrm{CH}_{3} \mathrm{OH}} \\
& \Leftrightarrow \underset{(\mathrm{DMA})}{\mathrm{C}_{4} \mathrm{H}_{8}\left(\mathrm{CH}_{3} \mathrm{COO}\right)_{2}}+\mathrm{H}_{2} \mathrm{O}
\end{aligned}
$$

Similarly two-step esterification also occurs in the synthesis of DMG. The major products from this series of reactions are water and DBE, which are partially miscible, and thus the product mixtures containing water, DBE, and alcohol may form two liquid phases in a certain range of compositions. The LLE data for such aqueous systems are fundamentally important for the process development. Among the previous related experimental works, Stepheson and Stuart [3] measured the mutual solubilities for various water-organic pairs, including water $+\mathrm{DMG}$, at temperatures from 273.15 to $363.15 \mathrm{~K}$. Uusi-Penttila et al. [1] determined experimentally the binodal curves and a few tie-lines for several ternary systems of DBE + water + solvent, such as DMA + MMA + water, at $297 \mathrm{~K}$. Ince and Kirbaslar $[2,4]$ and Ince $[5,6]$ investigated the LLE behavior of water + ethanol + DMG, water + ethanol + DMA, 
water + acetic acid + DMG, and water + acetic acid + DMA at 298.15, 308.15 and $318.15 \mathrm{~K}$.

The objective of this study is to measure the LLE data of water + methanol + DMA, water + MMA + DMA, and water + methanol+DMG at temperatures from 298.15 to $318.15 \mathrm{~K}$. These new experimental data were correlated with the NRTL model [7] and used to test the validity of the UNIFAC model [8] and its various modified versions, including UNIFAC-LLE [9] and UNIFAC-Dortmund [10].

\section{Experimental}

\subsection{Materials}

Methanol (99.9\%, Acros, USA), dimethyl adipate (DMA, 99+\%, Acros, USA), dimethyl glutarate (DMG, 98.5+\%, Acros, USA), adipic acid monomethyl ester (or monomethyl adipate, MMA, 99\%, Sigma-Aldrich, USA) were used in the present experiments without further purification. Deionized distilled water was prepared in our laboratory. The purity of the organic compounds has been checked with chromatographic analysis. While the peak area of impurity was detected to be $0.6 \%$ in DMG, trace amounts of impurities were found in other chemicals.

\subsection{Apparatus and procedures}

A glass-made LLE apparatus with an internal volume of about $10 \mathrm{~cm}^{3}$ was used in the present study. Its schematic diagram, as shown in Fig. 1, is similar to that of Peschke and Sandler [11]. Thermostatic water was circulated through the jacket of the equilibrium cell to control the cell's temperature to within $\pm 0.1 \mathrm{~K}$. The cell's temperature was measured with a precision thermometer (6, Model-1560, Hart Scientific Co., USA) with an uncertainty of $\pm 0.02 \mathrm{~K}$. About $10 \mathrm{~cm}^{3}$ of a liquid mixture, at a given total composition, was loaded in the equilibrium cell

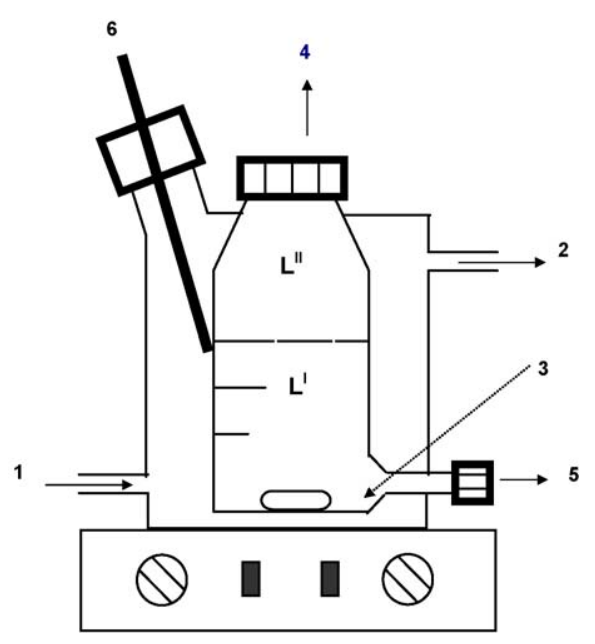

Fig. 1. Schematic diagram for LLE apparatus. (1) Thermostatic water inlet, (2) thermostatic water outlet, (3) stir bar, (4) sampling port for upper phase $\left(\mathrm{L}^{\mathrm{II}}\right)$, (5) sampling port for lower phase $\left(\mathrm{L}^{\mathrm{I}}\right)$, and (6) precision thermometer. from the top sampling port (4), and the port was then sealed by a screw open top cap with a Teflon septum. Before liquid mixtures loading, the bottom sampling port (5) has been sealed by another screw open top cap with a Teflon septum. A magnetic stirrer (3) vigorously agitated the liquid mixture in the cell. For each run, the contents were agitated longer than four hours to well mix the compounds. The mixture was allowed to settle at least $8 \mathrm{~h}$ to completely separate the two liquid phases. The phase compositions were found to remain unchanged as the settling periods were longer than $8 \mathrm{~h}$. A sample of upper liquid phase was taken with a syringe from the top sampling port (4) and that of lower liquid phase from the sampling port at the bottom of the cell (5) to avoid cross contamination by the other phase during the sampling process. The equilibrium compositions were determined from five replicated samples for each phase and an individual sample size was about $1 \mu l$. The phase compositions were analyzed by a gas chromatography (Model 8700, China Chromatography Co., Taiwan) with a thermal conductivity detector. A fused silica column CP-WAX 53CB $(0.53 \mathrm{~mm} \times 2 \mu \mathrm{m} \times 50 \mathrm{~m})$ was employed for separating the constituent compounds of samples. High purity helium (99.99\%) was used as a carrier gas. The averaged area fraction from the gas chromatography analysis was converted into mole fraction via calibration equations. Calibrations were made with gravimetrically prepared binary samples within two composition ranges, in accordance with those in the organic-rich phase and the water-rich phase for the ternary systems. An auxiliary solvent was added to homogenize the prepared standard samples for those constituent compounds, which were partilly miscible. The calibration was checked frequently to keep the accuracy of composition analysis. The uncertainties of composition analysis for the minor components are tabulated in Table 1.

\subsection{Experimental results}

The LLE apparatus was used to measure the tie-line data for the ternary systems of water + methanol + DMA, water + MMA + DMA, and water + methanol + DMG at temperatures from 298.15 to $318.15 \mathrm{~K}$. Tables $2-4$ list the compositions of two coexistence liquid phases for these three ternary systems, while the mutual solubilities of water/DMA

Table 1

Uncertainties of composition analysis

\begin{tabular}{lll}
\hline Mixture & Phase & AAD $\Delta x^{\mathrm{a}}$ \\
\hline Water + methanol & & 0.0017 \\
Water + MMA & & 0.0017 \\
Water + DMA & Organic & 0.0005 \\
& Aqueous & 0.0001 \\
Water + DMG & Organic & 0.0011 \\
& Aqueous & 0.0001 \\
\hline
\end{tabular}

${ }^{\text {a }} \mathrm{AAD} \Delta x=\left(1 / n_{\mathrm{P}}\right) \sum_{j=1}^{n_{\mathrm{P}}}\left(\left|x^{\text {calb }}-x^{\text {act }}\right|\right)_{j}$, where $n_{\mathrm{P}}$ is the number of calibration points, $x^{\text {act }}$ refers to the actual mole fraction of a prepared standard sample, and $x^{\text {calb }}$ is the corresponding mole fraction converted from the area fraction of gas chromatography analysis via the calibration equation. 
Table 2

LLE data for water (1) + methanol (2) + DMA (3) at atmospheric pressure

\begin{tabular}{|c|c|c|c|c|c|c|}
\hline \multirow[t]{2}{*}{$T(\mathrm{~K})$} & \multicolumn{3}{|c|}{ Organic phase } & \multicolumn{3}{|c|}{ Aqueous phase } \\
\hline & $x_{1}^{\mathrm{I}}$ & $x_{2}^{\mathrm{I}}$ & $x_{3}^{\mathrm{I}}$ & $x_{1}^{\mathrm{II}}$ & $x_{2}^{\mathrm{II}}$ & $x_{3}^{\mathrm{II}}$ \\
\hline \multirow{6}{*}{298.15} & 0.2555 & 0.0480 & 0.6965 & 0.9452 & 0.0478 & 0.0070 \\
\hline & 0.2693 & 0.0820 & 0.6487 & 0.9128 & 0.0818 & 0.0054 \\
\hline & 0.2923 & 0.1258 & 0.5819 & 0.8673 & 0.1255 & 0.0072 \\
\hline & 0.3157 & 0.1525 & 0.5318 & 0.8423 & 0.1491 & 0.0086 \\
\hline & 0.3728 & 0.2100 & 0.4172 & 0.7913 & 0.1954 & 0.0133 \\
\hline & 0.4360 & 0.2511 & 0.3129 & 0.7435 & 0.2319 & 0.0246 \\
\hline \multirow{6}{*}{308.15} & 0.2797 & 0.0482 & 0.6721 & 0.9464 & 0.0454 & 0.0082 \\
\hline & 0.2906 & 0.0847 & 0.6247 & 0.9172 & 0.0773 & 0.0055 \\
\hline & 0.3175 & 0.1296 & 0.5529 & 0.8749 & 0.1175 & 0.0076 \\
\hline & 0.3545 & 0.1586 & 0.4869 & 0.8504 & 0.1396 & 0.0100 \\
\hline & 0.4074 & 0.2103 & 0.3823 & 0.8027 & 0.1831 & 0.0142 \\
\hline & 0.4925 & 0.2504 & 0.2571 & 0.7402 & 0.2250 & 0.0348 \\
\hline \multirow{6}{*}{318.15} & 0.2991 & 0.0517 & 0.6492 & 0.9510 & 0.0431 & 0.0059 \\
\hline & 0.3126 & 0.0864 & 0.6010 & 0.9223 & 0.0719 & 0.0058 \\
\hline & 0.3474 & 0.1311 & 0.5215 & 0.8820 & 0.1103 & 0.0077 \\
\hline & 0.3757 & 0.1565 & 0.4678 & 0.8589 & 0.1310 & 0.0101 \\
\hline & 0.4267 & 0.2078 & 0.3655 & 0.8098 & 0.1751 & 0.0151 \\
\hline & 0.5412 & 0.2493 & 0.2095 & 0.7300 & 0.2197 & 0.0503 \\
\hline
\end{tabular}

and water/DMG have been available from Ince [6] and Stepheson and Stuart [3]. Fig. 2 compares the experimental results of water + MMA + DMA at $298.15 \mathrm{~K}$ with the LLE data at $297 \mathrm{~K}$ reported by Uusi-Penttila et al. [1]. The comparison shows that the agreement is reasonably well. The experimental LLE phase envelopes obtained from the present study are illustrated in

Table 3

LLE data for water (1) + MMA (2) + DMA (3) at atmospheric pressure

\begin{tabular}{|c|c|c|c|c|c|c|}
\hline \multirow[t]{2}{*}{$T(\mathrm{~K})$} & \multicolumn{3}{|c|}{ Organic phase } & \multicolumn{3}{|c|}{ Aqueous phase } \\
\hline & $x_{1}^{\mathrm{I}}$ & $x_{2}^{\mathrm{I}}$ & $x_{3}^{\mathrm{I}}$ & $x_{1}^{\mathrm{II}}$ & $x_{2}^{\mathrm{II}}$ & $x_{3}^{\mathrm{II}}$ \\
\hline \multirow{8}{*}{298.15} & 0.2608 & 0.0269 & 0.7123 & 0.9948 & 0.0008 & 0.0044 \\
\hline & 0.2822 & 0.0435 & 0.6743 & 0.9956 & 0.0010 & 0.0034 \\
\hline & 0.3022 & 0.0600 & 0.6378 & 0.9956 & 0.0015 & 0.0029 \\
\hline & 0.3549 & 0.0939 & 0.5512 & 0.9935 & 0.0027 & 0.0038 \\
\hline & 0.3833 & 0.1099 & 0.5068 & 0.9934 & 0.0029 & 0.0037 \\
\hline & 0.4102 & 0.1258 & 0.4640 & 0.9930 & 0.0035 & 0.0035 \\
\hline & 0.4721 & 0.1537 & 0.3742 & 0.9894 & 0.0060 & 0.0046 \\
\hline & 0.4974 & 0.1719 & 0.3307 & 0.9853 & 0.0076 & 0.0071 \\
\hline \multirow{8}{*}{308.15} & 0.2893 & 0.0252 & 0.6855 & 0.9958 & 0.0007 & 0.0035 \\
\hline & 0.3060 & 0.0432 & 0.6508 & 0.9954 & 0.0012 & 0.0034 \\
\hline & 0.3294 & 0.0616 & 0.6090 & 0.9953 & 0.0015 & 0.0032 \\
\hline & 0.3740 & 0.0917 & 0.5343 & 0.9941 & 0.0025 & 0.0034 \\
\hline & 0.3960 & 0.1070 & 0.4970 & 0.9939 & 0.0029 & 0.0032 \\
\hline & 0.4304 & 0.1211 & 0.4485 & 0.9925 & 0.0036 & 0.0039 \\
\hline & 0.4827 & 0.1531 & 0.3642 & 0.9896 & 0.0057 & 0.0047 \\
\hline & 0.5104 & 0.1642 & 0.3254 & 0.9889 & 0.0066 & 0.0045 \\
\hline \multirow{8}{*}{318.15} & 0.3117 & 0.0229 & 0.6654 & 0.9950 & 0.0014 & 0.0036 \\
\hline & 0.3240 & 0.0421 & 0.6339 & 0.9956 & 0.0011 & 0.0033 \\
\hline & 0.3428 & 0.0586 & 0.5986 & 0.9936 & 0.0020 & 0.0044 \\
\hline & 0.3924 & 0.0889 & 0.5187 & 0.9928 & 0.0029 & 0.0043 \\
\hline & 0.4168 & 0.1054 & 0.4778 & 0.9916 & 0.0035 & 0.0049 \\
\hline & 0.4558 & 0.1195 & 0.4247 & 0.9898 & 0.0046 & 0.0056 \\
\hline & 0.5114 & 0.1469 & 0.3417 & 0.9880 & 0.0068 & 0.0052 \\
\hline & 0.5405 & 0.1591 & 0.3004 & 0.9867 & 0.0049 & 0.0084 \\
\hline
\end{tabular}

Table 4

LLE data for water (1) + methanol (2) + DMG (3) at atmospheric pressure

\begin{tabular}{|c|c|c|c|c|c|c|}
\hline \multirow[t]{2}{*}{$T(\mathrm{~K})$} & \multicolumn{3}{|c|}{ Organic phase } & \multicolumn{3}{|c|}{ Aqueous phase } \\
\hline & $x_{1}^{\mathrm{I}}$ & $x_{2}^{\mathrm{I}}$ & $x_{3}^{\mathrm{I}}$ & $x_{1}^{\mathrm{II}}$ & $x_{2}^{\mathrm{II}}$ & $x_{3}^{\mathrm{II}}$ \\
\hline \multirow{7}{*}{298.15} & 0.2637 & 0.0347 & 0.7016 & 0.9546 & 0.0375 & 0.0079 \\
\hline & 0.2901 & 0.0563 & 0.6536 & 0.9353 & 0.0551 & 0.0096 \\
\hline & 0.3016 & 0.0737 & 0.6247 & 0.9107 & 0.0776 & 0.0117 \\
\hline & 0.3187 & 0.0982 & 0.5831 & 0.8849 & 0.1010 & 0.0141 \\
\hline & 0.3469 & 0.1216 & 0.5315 & 0.8523 & 0.1321 & 0.0156 \\
\hline & 0.4035 & 0.1628 & 0.4337 & 0.8032 & 0.1769 & 0.0199 \\
\hline & 0.4352 & 0.1851 & 0.3797 & 0.7603 & 0.2158 & 0.0239 \\
\hline \multirow{7}{*}{308.15} & 0.2939 & 0.0371 & 0.6690 & 0.9559 & 0.0359 & 0.0082 \\
\hline & 0.3104 & 0.0552 & 0.6344 & 0.9379 & 0.0520 & 0.0101 \\
\hline & 0.3323 & 0.0787 & 0.5890 & 0.9171 & 0.0717 & 0.0112 \\
\hline & 0.3560 & 0.1036 & 0.5404 & 0.8898 & 0.0962 & 0.0140 \\
\hline & 0.3799 & 0.1290 & 0.4911 & 0.8597 & 0.1210 & 0.0193 \\
\hline & 0.4354 & 0.1668 & 0.3978 & 0.8372 & 0.1371 & 0.0257 \\
\hline & 0.4847 & 0.1872 & 0.3281 & 0.7724 & 0.2027 & 0.0249 \\
\hline \multirow{7}{*}{318.15} & 0.3162 & 0.0388 & 0.6450 & 0.9574 & 0.0339 & 0.0087 \\
\hline & 0.3351 & 0.0564 & 0.6085 & 0.9397 & 0.0493 & 0.0110 \\
\hline & 0.3581 & 0.0808 & 0.5611 & 0.9205 & 0.0674 & 0.0121 \\
\hline & 0.3870 & 0.1066 & 0.5064 & 0.8957 & 0.0882 & 0.0161 \\
\hline & 0.4127 & 0.1340 & 0.4533 & 0.8689 & 0.1134 & 0.0177 \\
\hline & 0.4526 & 0.1624 & 0.3850 & 0.8259 & 0.1538 & 0.0203 \\
\hline & 0.4907 & 0.1799 & 0.3294 & 0.7799 & 0.1908 & 0.0293 \\
\hline
\end{tabular}

Figs. 3-5 for water + methanol + DMA, water + MMA + DMA, and water + methanol + DMG, respectively. Since methanol and MMA are completely miscible with water, these three ternary systems behave as type-I LLE. As seen from Fig. 3, the solubility of DMA is very sparing in the aqueous phase, while water dissolves appreciably in the organic-rich phase. Also the area of liquid phase splitting zone enlarges as temperature decreases. Fig. 4 shows the LLE phase diagram of water + MMA + DMA over the experimental conditions. In this ternary system, the solubilities of both DMA and MMA in the aqueous phase are so minute that the aqueous phase ends of the tie-lines are located closely to the

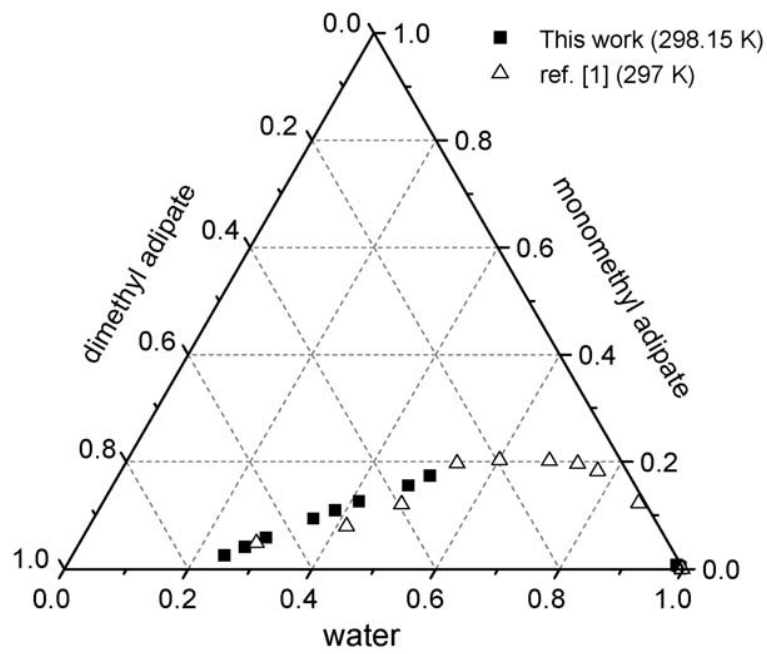

Fig. 2. Comparison of experimental results with literature values (Ref. [1]) for water + MMA + DMA. Each coordinate variable is in mole fraction. 


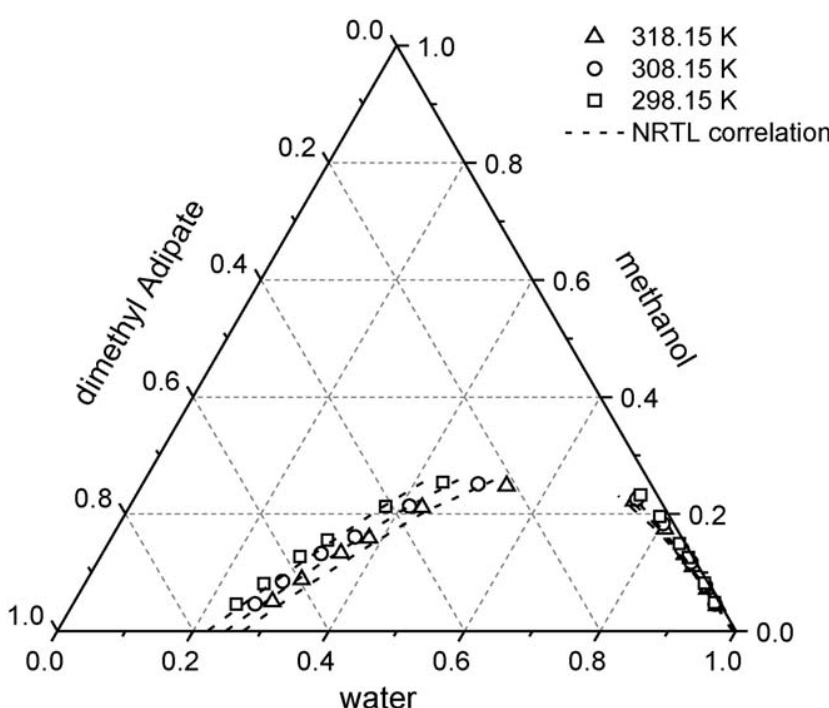

Fig. 3. LLE experimental and correlated results for water + methanol + DMA. Each coordinate variable is in mole fraction.

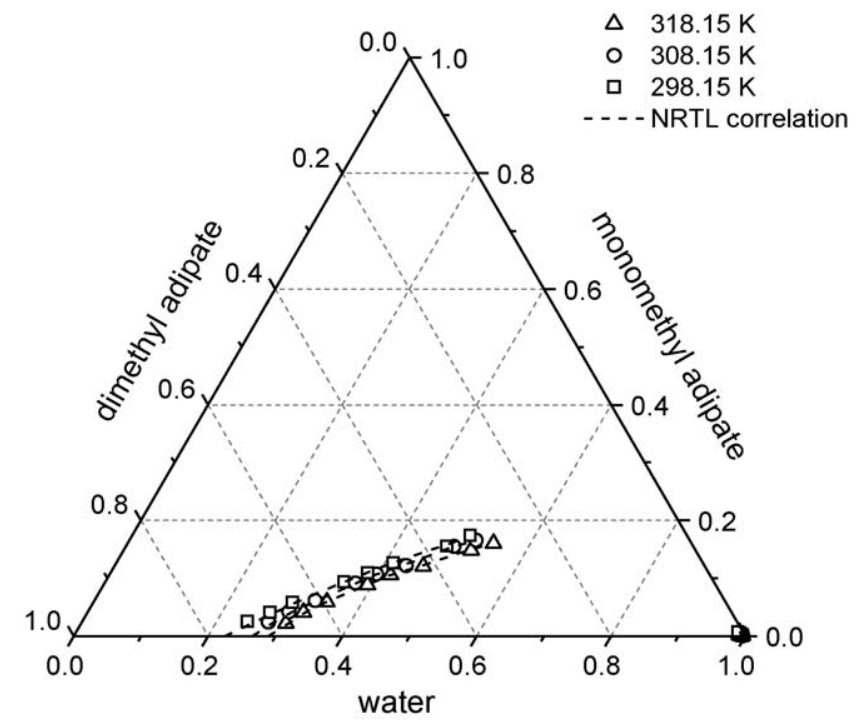

Fig. 4. LLE experimental and correlated results for water + MMA + DMA. Each coordinate variable is in mole fraction.

corner of pure water. Fig. 5 presents the LLE phase boundaries of water + methanol + DMG from 298.15 to $318.15 \mathrm{~K}$. Its phase behavior is similar to that of water + methanol + DMA, but the solubilities of methanol in both two liquid phases are relatively lower in this ternary system.

\section{Phase equilibrium calculations}

Reliable thermodynamic models are essentially needed in process simulation and design. The new phase equilibrium data obtained from the present study form a basis for thermodynamic models verification and parameters determination. At liquid-liquid equilibrium, the compositions of two coexistence liquid phases can be calculated from the criteria of LLE (equality of the constituent fugacities between the coexistence phases) together with material balance equation. The calculation proce-

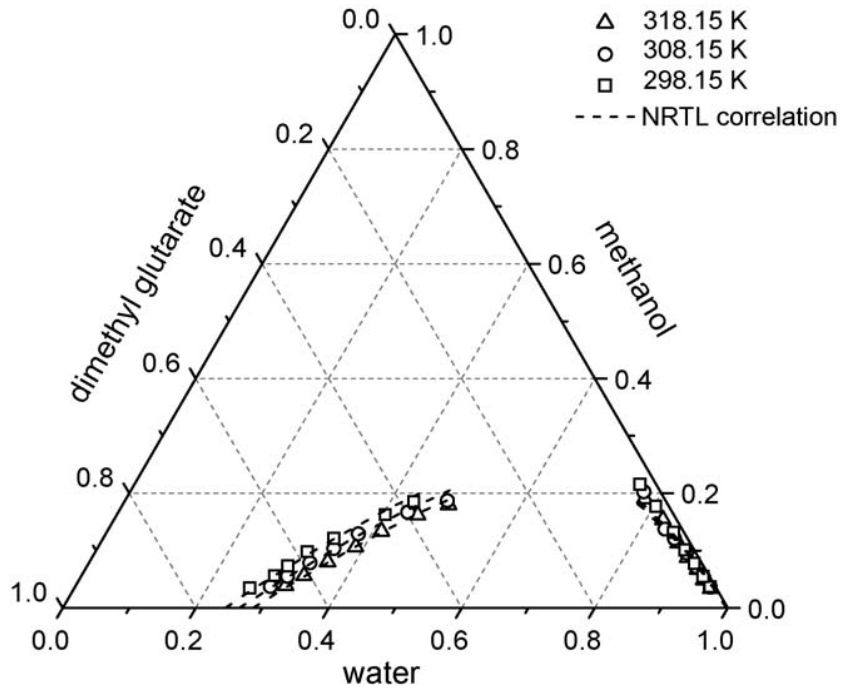

Fig. 5. LLE experimental and correlated results for water + methanol + DMG. Each coordinate variable is in mole fraction.

dure has been detailed in Walas [12]. Based on one mole of feed with total composition $z_{i}$, the material balance and equilibrium equations for component $i$ are given as followed:

$z_{i}=\beta x_{i}^{\mathrm{I}}+(1-\beta) x_{i}^{\mathrm{II}}$

and

$K_{i}=\frac{x_{i}^{\mathrm{II}}}{x_{i}^{\mathrm{I}}}=\frac{\gamma_{i}^{\mathrm{I}}}{\gamma_{i}^{\mathrm{II}}}$

where $\beta$ is the fraction of the lower liquid phase (the first liquid phase), $K_{i}$ and $\gamma_{i}$ the distribution ratio and the activity coefficient for component $i$, and the superscripts I and II represent the lower and upper liquid phases, respectively. From Eqs. (2) and (3), the mole fraction of component $i$ in the first liquid phase $x_{i}^{\mathrm{I}}$ can be expressed as

$x_{i}^{\mathrm{I}}=\frac{z_{i}}{\beta+K_{i}(1-\beta)}$

Introducing the above equation into the constraint of mole fractions for the first liquid phase yields the liquid phase splitting equation:

$f(\beta)=1-\sum_{i=1}^{n_{\mathrm{c}}} x_{i}^{\mathrm{I}}=1-\sum_{i=1}^{n_{c}} \frac{z_{i}}{\beta+K_{i}(1-\beta)}=0$

where $n_{\mathrm{c}}$ is the number of components. After the value of $\beta$ has been solved iteratively, the compositions of the coexistence liquid phases were obtained from Eqs. (4) and (3).

The new experimental data were firstly used here to examine the validity of several versions of the groupcontribution based models, including the original UNIFAC model [8], the UNIFAC-LLE [9] and the UNIFAC-Dortmund [10] (denoted as UNIFAC-DMD). The predicted results from these three versions of the UNIFAC are summarized in Table 5. Figs. 6-8 compare the predicted phase envelops from the different versions of UNIFAC with the experimental 


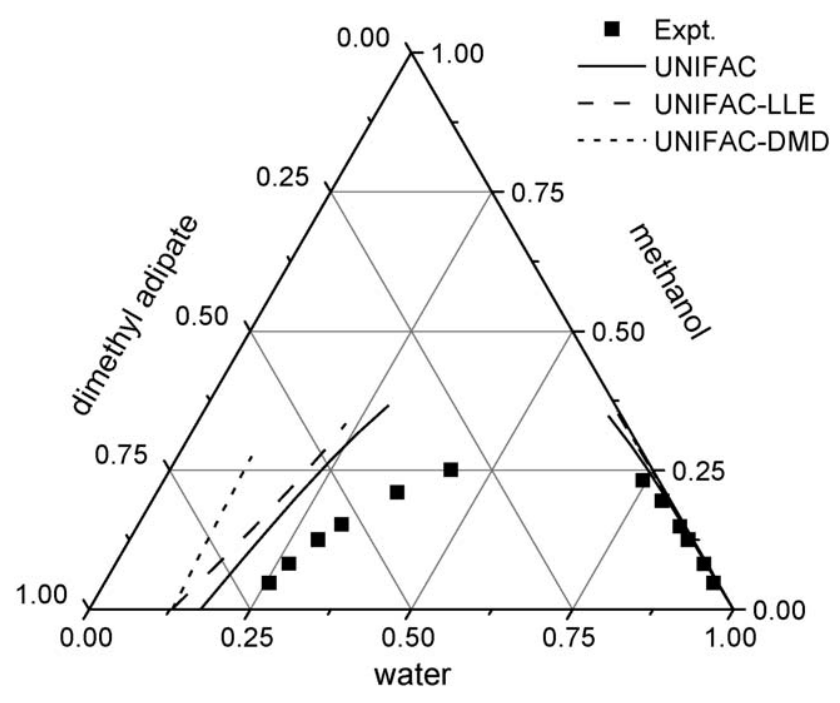

Fig. 6. Comparison of the predictions from the UNIFAC models with experimental results for water + methanol + DMA at $298.15 \mathrm{~K}$.

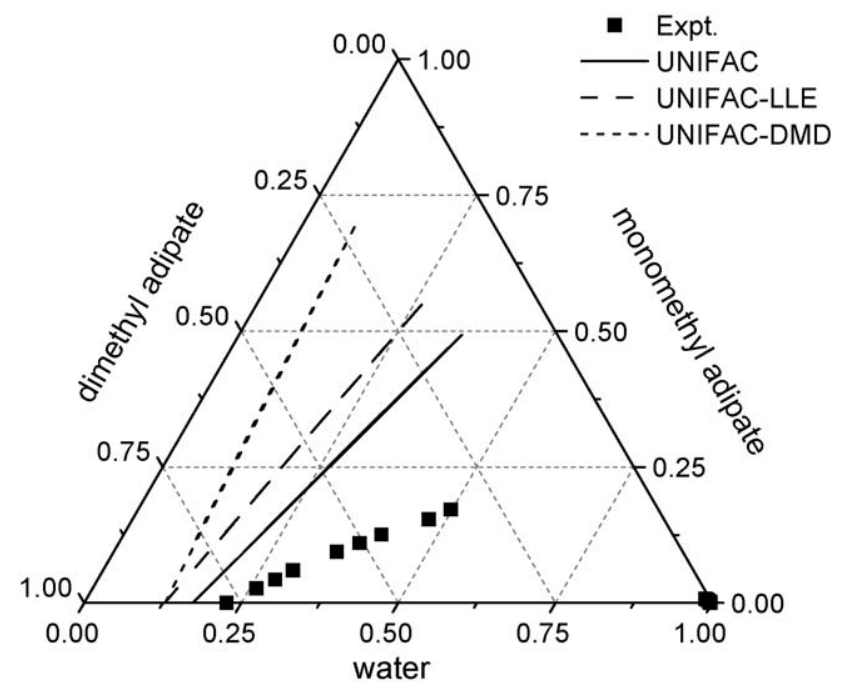

Fig. 7. Comparison of the predictions from the UNIFAC models with experimental results for water + MMA + DMA at 298.15 K.

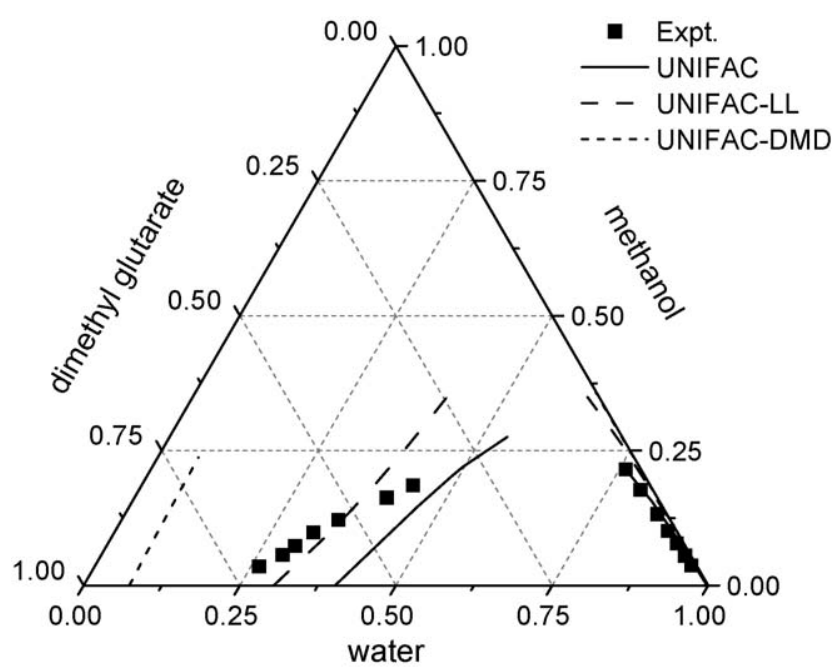

Fig. 8. Comparison of the predictions from the UNIFAC models with experimental results for water + methanol + DMG at 298.15 K.
Table 5

Predicted results from three versions of UNIFAC models

\begin{tabular}{lllll}
\hline Model & Phase & \multicolumn{3}{l}{ Grand AAD $\Delta x^{\mathrm{a}}$} \\
\cline { 3 - 5 } & & $298.15 \mathrm{~K}$ & $308.15 \mathrm{~K}$ & $318.15 \mathrm{~K}$ \\
\hline Water + methanol + DMA & & & \\
UNI- & Organic & 0.0659 & 0.0805 & 0.0894 \\
FAC & Aqueous & 0.0286 & 0.0291 & 0.0300 \\
UNIFAC- & Organic & 0.1143 & 0.0992 & 0.1074 \\
LLE & Aqueous & 0.0956 & 0.0298 & 0.0305 \\
UNIFAC- & Organic & 0.1225 & 0.1346 & 0.1401 \\
DMD & Aqueous & 0.0361 & 0.0361 & 0.0365 \\
Water + MMA + DMA & & & \\
UNI- & Organic & 0.0861 & 0.0874 & 0.1060 \\
FAC & Aqueous & 0.0034 & 0.0029 & 0.0038 \\
UNIFAC- & Organic & 0.1198 & 0.1154 & 0.1383 \\
LLE & Aqueous & 0.0037 & 0.0032 & 0.0042 \\
UNIFAC- & Organic & 0.1360 & 0.1293 & 0.1484 \\
DMD & Aqueous & 0.0036 & 0.0031 & 0.0041 \\
Water + methanol + DMG & & & \\
UNI- & Organic & 0.0939 & 0.0720 & 0.0610 \\
FAC & Aqueous & 0.0243 & 0.0181 & 0.0176 \\
UNIFAC- & Organic & 0.0370 & 0.0327 & 0.0307 \\
LLE & Aqueous & 0.0158 & 0.0124 & 0.0108 \\
UNIFAC- & Organic & 0.1837 & 0.1955 & 0.1953 \\
DMD & Aqueous & 0.0104 & 0.0139 & 0.0129 \\
\hline
\end{tabular}

${ }^{\text {a }}$ Grand AAD $\Delta x=\left[\sum_{k=1}^{n_{\mathrm{TL}}} \sum_{j=1}^{n_{\mathrm{L}}} \sum_{i=1}^{n_{\mathrm{c}}}\left|x_{i, k}^{j, \text { calc }}-x_{i, k}^{j, \text { expt }}\right|\right] / 6 n_{\mathrm{TL}}, \quad$ where $n_{\mathrm{TL}}, n_{\mathrm{L}}$, and $n_{\mathrm{c}}$ are the number of tie-lines, the number of phases, and the number of components, respectively.

data for water + methanol + DMA, water + MMA + DMA, and water + methanol + DMG at $298.15 \mathrm{~K}$, respectively. The UNIFAC models appear to obviously overestimate the regions of heterogeneity for water + methanol + DMA and water + MMA + DMA, as shown in Figs. 6 and 7, respectively. Fig. 8 also shows that all versions of the UNIFAC fail to accurately predict the binodal curves for water + methanol + DMG, especially in the region near the plait point. A correlative activity coefficient model is necessary to quantitatively represent the phase behavior of these investigated systems.

The NRTL model was thus applied in the present study to correlate the new LLE data. Temperature-dependent NRTL parameters were used for representing the phase behavior over the entire experimental temperature range. The definition of the NRTL model is given as following:

$$
\begin{aligned}
\ln \gamma_{i}= & \frac{\sum_{j=1}^{n_{c}} x_{j} \tau_{i j} G_{j i}}{\sum_{k=1}^{n_{c}} x_{k} G_{k i}} \\
& +\sum_{j=1}^{n_{c}} \frac{x_{j} G_{i j}}{\sum_{k=1}^{n} x_{k} G_{k j}}\left(\tau_{i j}-\frac{\sum_{m=1}^{n_{c}} x_{m} \tau_{m j} G_{m j}}{\sum_{k=1}^{n_{c}} x_{k} G_{k j}}\right)
\end{aligned}
$$

where $G_{i j}=\exp \left(-\alpha_{i j} \tau_{i j}\right)$ and $\tau_{i j}=a_{i j}+b_{i j} / T$. There are three adjustable parameters, $a_{i j}, b_{i j}$, and $\alpha_{i j}$, for each constituent binary. The optimal values of the NRTL parameters were deter- 
Table 6

Correlated results from the NRTL model for water (1) + methanol (2) + DMA (3)

\begin{tabular}{llllll}
\hline$i-j$ & $\alpha_{i j}$ & $a_{i j}$ & \multicolumn{1}{l}{$a_{j i}$} & \multicolumn{1}{l}{$b_{i j}(\mathrm{~K})$} & \multicolumn{1}{c}{$b_{j i}(\mathrm{~K})$} \\
\hline $1-2$ & 0.3 & 4.868 & -2.631 & -1830.863 & 306.944 \\
$1-3$ & 0.2936 & 4.039 & -2.995 & 218.692 & 1160.826 \\
$2-3$ & 0.3 & 0.549 & -2.988 & 452.196 & -108.168 \\
& & & & & \\
$T(\mathrm{~K})$ & Phase & AAD $\Delta x_{1}{ }^{\mathrm{a}}$ & $\mathrm{AAD} \Delta x_{2}{ }^{\mathrm{a}}$ & $\mathrm{AAD} \Delta x_{3}{ }^{\mathrm{a}}$ & \multicolumn{1}{c}{ Grand } \\
& & & & & $\mathrm{AAD} \Delta x^{\mathrm{b}}$ \\
\hline \multirow{2}{*}{298.15} & Organic & 0.0074 & 0.0053 & 0.0057 & \\
& Aqueous & 0.0068 & 0.0056 & 0.0064 & 0.0062 \\
308.15 & Organic & 0.0118 & 0.0068 & 0.0071 & \\
& Aqueous & 0.0082 & 0.0042 & 0.0067 & 0.0074 \\
318.15 & Organic & 0.0148 & 0.0089 & 0.0076 & \\
& Aqueous & 0.0077 & 0.0052 & 0.0068 & 0.0085 \\
\hline
\end{tabular}

${ }^{\text {a }} \mathrm{AAD} \Delta x_{i}=\left[\sum_{k=1}^{n_{\mathrm{TL}}}\left|x_{i, k}^{\mathrm{calc}}-x_{i, k}^{\text {expt }}\right|\right] / n_{\mathrm{TL}}$, where $n_{\mathrm{TL}}$ and $n_{\mathrm{c}}$ are the number of tie-lines and the number of components, respectively.

${ }^{\mathrm{b}}$ Grand AAD $\Delta x=\left[\sum_{k=1}^{n_{\mathrm{TL}}} \sum_{j=1}^{n_{\mathrm{L}}} \sum_{i=1}^{n_{\mathrm{c}}}\left|x_{i, k}^{j, \text { calc }}-x_{i, k}^{j, \text { expt }}\right|\right] / 6 n_{\mathrm{TL}}, \quad$ where $n_{\mathrm{TL}}, n_{\mathrm{L}}$, and $n_{\mathrm{c}}$ are the number of tie-lines, the number of phases, and the number of components, respectively.

mined by minimization of the following objective function $(\pi)$ :

$\pi=\sum_{k=1}^{n_{\mathrm{TL}}} \sum_{j=1}^{n_{\mathrm{L}}} \sum_{i=1}^{n_{\mathrm{c}}}\left(x_{i, k}^{j, \text { calc }}-x_{i, k}^{j, \text { expt }}\right)^{2}$

where $n_{\mathrm{TL}}, n_{\mathrm{L}}$, and $n_{\mathrm{c}}$ are the number of tie-lines, the number of phases, and the number of components, respectively. Tables 6-8 report the correlated results for the ternary systems of water + methanol + DMA, water + MMA + DMA, and water + methanol + DMG, respectively. The calculated binodal curves (denoted as NRTL correlation) are compared with the experimental values in Figs. 3-5, indicating that the correlated results from the NRTL model agree satisfactorily with the experimental values over the entire experimental conditions. These

Table 7

Correlated results from the NRTL model for water (1) + MMA (2) + DMA (3)

\begin{tabular}{llllll}
\hline$i-j$ & $\alpha_{i j}$ & $a_{i j}$ & $a_{j i}$ & \multicolumn{1}{c}{$b_{i j}(\mathrm{~K})$} & \multicolumn{1}{c}{$b_{j i}(\mathrm{~K})$} \\
\hline $1-2$ & 0.6658 & 11.329 & -1.526 & -2476.828 & 373.602 \\
$1-3$ & 0.2936 & 4.039 & -2.995 & 218.692 & 1160.826 \\
$2-3$ & 0.3 & 0 & 0 & -540.931 & 1416.636 \\
& & & & & \\
$T(\mathrm{~K})$ & Phase & AAD $\Delta x_{1}{ }^{\mathrm{a}}$ & $\mathrm{AAD} \Delta x_{2}{ }^{\mathrm{a}}$ & $\mathrm{AAD} \Delta x_{3}{ }^{\mathrm{a}}$ & Grand \\
& & & & & $\mathrm{AAD} \Delta x^{\mathrm{b}}$ \\
\hline \multirow{2}{*}{298.15} & Organic & 0.0004 & 0.0007 & 0.0004 & 0.0009 \\
& Aqueous & 0.0021 & 0.0013 & 0.0010 & \\
308.15 & Organic & 0.0006 & 0.0006 & 0.0005 & 0.0009 \\
& Aqueous & 0.0018 & 0.0009 & 0.0011 & \\
318.15 & Organic & 0.0007 & 0.0009 & 0.0006 & 0.0010 \\
& Aqueous & 0.0020 & 0.0008 & 0.0013 & \\
\hline
\end{tabular}

a $\mathrm{AAD} \Delta x_{i}=\left[\sum_{k=1}^{n \mathrm{TL}}\left|x_{i, k}^{\text {calc }}-x_{i, k}^{\text {expt }}\right|\right] / n_{\mathrm{TL}}$, where $n_{\mathrm{TL}}$ and $n_{\mathrm{c}}$ are the number of tie-lines and the number of components, respectively.

${ }^{\mathrm{b}}$ Grand AAD $\Delta x=\left[\sum_{k=1}^{n_{\mathrm{TL}}} \sum_{j=1}^{n_{\mathrm{L}}} \sum_{i=1}^{n_{\mathrm{c}}}\left|x_{i, k}^{j, \text { calc }}-x_{i, k}^{j, \text { expt }}\right|\right] / 6 n_{\mathrm{TL}}$, where $n_{\mathrm{TL}}, n_{\mathrm{L}}$, and $n_{\mathrm{c}}$ are the number of tie-lines, the number of phases, and the number of components, respectively.
Table 8

Correlated results from the NRTL model for water (1) + methanol (2) + DMG (3)

\begin{tabular}{llllll}
\hline$i-j$ & $\alpha_{i j}$ & \multicolumn{1}{c}{$a_{i j}$} & \multicolumn{1}{l}{$a_{j i}$} & \multicolumn{1}{c}{$b_{i j}(\mathrm{~K})$} & \multicolumn{1}{c}{$b_{j i}(\mathrm{~K})$} \\
\hline $1-2^{\mathrm{a}}$ & 0.3 & 4.868 & -2.631 & -1830.863 & 306.944 \\
$1-3$ & 0.3 & 3.523 & -2.562 & 600.310 & 1054.101 \\
$2-3$ & 0.2 & -0.468 & -5.180 & 395.060 & 688.767 \\
& & & & & \\
$T(\mathrm{~K})$ & Phase & $\mathrm{AAD} \Delta x_{1}{ }^{\mathrm{a}}$ & $\mathrm{AAD} \Delta x_{2}{ }^{\mathrm{a}}$ & $\mathrm{AAD} \Delta x_{3}{ }^{\mathrm{a}}$ & \multicolumn{1}{l}{ Grand } \\
& & & & & $\mathrm{AAD} \Delta x^{\mathrm{b}}$ \\
\hline \multirow{2}{*}{298.15} & Organic & 0.0021 & 0.0060 & 0.0051 & 0.0052 \\
& Aqueous & 0.0034 & 0.0082 & 0.0065 & \\
308.15 & Organic & 0.0024 & 0.0047 & 0.0048 & 0.0050 \\
& Aqueous & 0.0058 & 0.0071 & 0.0055 & \\
318.15 & Organic & 0.0012 & 0.0041 & 0.0041 & 0.0039 \\
& Aqueous & 0.0023 & 0.0066 & 0.0051 & \\
\hline
\end{tabular}

${ }^{\text {a }}$ AAD $\Delta x_{i}=\left[\sum_{k=1}^{n_{\mathrm{TL}}}\left|x_{i, k}^{\mathrm{calc}}-x_{i, k}^{\mathrm{expt}}\right|\right] / n_{\mathrm{TL}}$, where $n_{\mathrm{TL}}$ and $n_{\mathrm{c}}$ are the number of tie-lines and the number of components, respectively.

${ }^{\mathrm{b}}$ Grand AAD $\Delta x=\left[\sum_{k=1}^{n_{\mathrm{TL}}} \sum_{j=1}^{n_{\mathrm{L}}} \sum_{i=1}^{n_{\mathrm{c}}}\left|x_{i, k}^{j, \text { calc }}-x_{i, k}^{j, \text { expt }}\right|\right] / 6 n_{\mathrm{TL}}$, where $n_{\mathrm{TL}}, n_{\mathrm{L}}$, and $n_{\mathrm{c}}$ are the number of tie-lines, the number of phases, and the number of components, respectively.

determined parameters can be applied to simulate operation of a decanter in the dibasic acids recovery process with reactive distillation method.

\section{Conclusions}

Liquid-liquid equilibrium data have been measured for three ternary systems of water + methanol + DMA, water + MMA + DMA, and water + methanol + DMG at temperatures ranging from 298.15 to $318.15 \mathrm{~K}$ under atmospheric pressure. All these three ternary systems behave as type-I LLE and the areas of the liquid-liquid splitting zone decrease with an increase of temperature. While the solubilities of water are appreciable in the organic-rich phase, those of the dibasic esters are very minute in the aqueous phase. Especially in the ternary system of water + MMA + DMA, the mole fractions of water are greater than 0.985 in the aqueous phase over the entire range of the experimental conditions. Moreover, this study found that various versions of UNIFAC models failed to reasonably predict the liquid-liquid phase boundaries for the investigated systems. The new LLE data can be accurately correlated with the NRTL model by using temperature-dependent binary interaction parameters. The NRTL model with these determined parameters provide a reliable basis to simulate the operation of a decanter, which is an essential unit in the dibasic acids recovery process with reactive distillation technique.

List of symbols

$a_{i j} \quad$ parameters of the NRTL model

AA adipic acid

AAD average absolute deviation

$b_{i j} \quad$ parameters of the NRTL model (K)

DBE dibasic esters

DMA dimethyl adipate

DMG dimethyl glutarate 
DMS dimethyl succinate

$K_{i} \quad$ distribution ratio for component $i$

$\mathrm{MeOH}$ methanol

MMA adipic acid monomethyl ester or monomethyl adipate

$n_{\mathrm{c}} \quad$ number of components

$n_{\mathrm{L}} \quad$ number of phases

$n_{\mathrm{P}} \quad$ number of calibration points

$n_{\mathrm{TL}} \quad$ number of tie-lines

$T \quad$ temperature (K)

$x \quad$ mole fraction in liquid phase

$z \quad$ mole fraction in feed

\section{Greek letters}

$\alpha_{i j} \quad$ non-randomness parameter of the NRTL model

$\beta \quad$ fraction of the total material in the first liquid phase

$\gamma_{i} \quad$ activity coefficient for component $i$

$\pi \quad$ objective function

$\tau_{i j} \quad$ parameters of the NRTL model

\section{Subscripts}

$\begin{array}{ll}i & \text { component } i \\ i j & i-j \text { pair interaction }\end{array}$

\section{Superscripts}

act actual value

calb calibrated value

calc calculated value
expt experimental value
I the first liquid phase (the lower liquid phase)
II the second liquid phase (the upper liquid phase)

\section{Acknowledgement}

Financial support from the Ministry of Economic Affairs, Taiwan, through Grant no. 94-EC-17-A-09-S1-019 is gratefully acknowledged.

\section{References}

[1] M. Uusi-Penttila, R.J. Richards, P. Blowers, B.A. Torgerson, K.A. Berglund, J. Chem. Eng. Data 41 (1996) 235-238.

[2] E. Ince, S.I. Kırbaslar, J. Chem. Thermodyn. 35 (2003) 1671-1679.

[3] R. Stepheson, J. Stuart, J. Chem. Eng. Data 31 (1986) 56-70.

[4] E. Ince, S.I. Kirbaslar, J. Chem. Eng. Data 50 (2005) 1539-1542.

[5] E. Ince, J. Chem. Eng. Data 50 (2005) 1539-1542.

[6] E. Ince, Fluid Phase Equilibria 230 (2005) 58-63.

[7] H. Renon, J.M. Prausnitz, AIChE J. 14 (1968) 135-144.

[8] A. Fredenslund, J. Gmehling, P. Rasmussen, Vapor-Liquid Equilibria Using UNIFAC: A Group-Contribution Method, Elsevier, Amsterdam, 1977.

[9] T. Magnussen, P. Rasmussen, A. Fredenslund, Ind. Eng. Chem. Process Des. Dev. 20 (1981) 331-339.

[10] J. Gmehling, J. Li, M. Schiller, S. Lee, Ind. Eng. Chem. Res. 32 (1993) 178-193.

[11] N. Peschke, S.I. Sandler, J. Chem. Eng. Data 40 (1995) 315-320.

[12] S.M. Walas, Phase Equilibria in Chemical Engineering, Butterworth, Boston, MA, 1985 